\title{
Comparación de los modelos de atención primaria en salud desde un enfoque sanitario en Colombia y sus países \\ fronterizos
}

A comparison of primary health care models from a health approach in Colombia and its limit-

ing countries

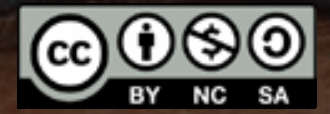

Daniela Sepúlveda Correa José Rafael Montaña Vásquez Martha Liliana Vargas Guette

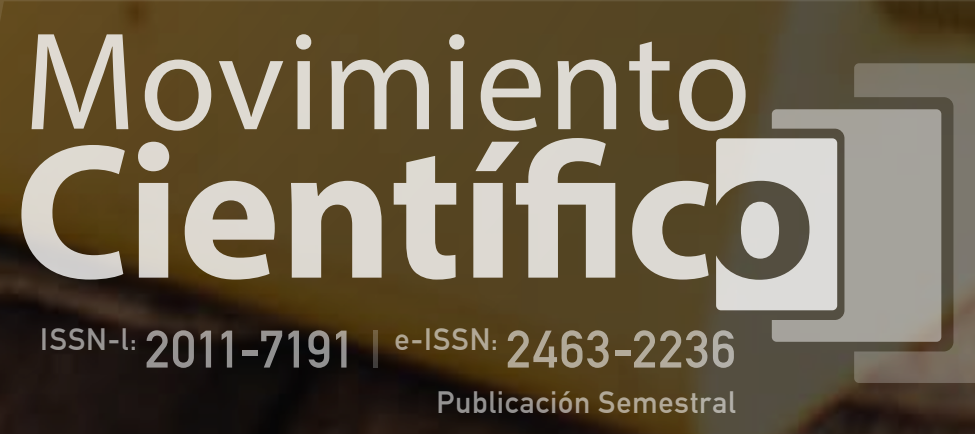


Movimiento Cientifico

ISSN-l: 2011-7191 | e-ISSN: 2463-2236 Publicación Semestral

ID: 10.33881/2011-7197.mct.15107

A comparison of primary health care models from a health approach in Colombia and its limiting countries

Título: Comparación de los modelos de atención primaria en salud desde un enfoque sanitario en Colombia y sus países fronterizos

Alt Title / Título alternativo:

A comparison of primary health care models from a health approach in Colombia and its limiting countries

[es]: $\quad$ Comparación de los modelos de atención primaria en salud desde un enfoque sanitario en Colombia y sus países fronterizos

Author (s) / Autor (es):

Sepúlveda Correa, Montaña Vásquez \& Vargas Guette

Keywords / Palabras Clave:

[en]: $\quad$ Primary Health Care, Millennium Development Goals, Public Health, Health systems

[es]: $\quad$ Atención Primaria en Salud, Objetivos del Desarrollo del Milenio, Salud pública, sistemas de salud

Submited: 2021-07-13

\section{Resumen}

Comparar los modelos de Atención Primaria en Salud de Colombia y sus países fronterizos con datos del año 2018, teniendo como base, indicadores de resultado de los Objetivos del Desarrollo del Milenio relacionados con la salud.Conclusiones: De los países analizados en este estudio, solamente Perú aplica políticas encaminadas al cumplimiento de estos indicadores y dichas políticas tienen un impacto positivo dentro del desarrollo de los objetivos en cada uno de ellos, siendo el país con mayor índice de cumplimiento.

En Venezuela fue donde se hallaron los mayores inconvenientes en cuanto a la información disponible ya que la situación política actual del país representa un hermetismo importante en la información pública relacionada con en el cumplimiento de los Objetivos del Desarrollo del Milenio relacionados con la salud en los indicadores específicos analizados, sin embargo, se ogró identificar que, en cuanto a políticas, únicamente

se cumplen los objetivos 5 y 6 pero teniendo un impacto negativo e irrelevante para cada uno de

ellos. En relación con el resto de estos, no se aplican políticas con el consecuente impacto negativo. Palabras claves: Atención Primaria en Salud, Objetivos del Desarrollo del Milenio, Salud pública, sistemas de

salud Materiales y métodos: Para la recolección de datos se utilizó una matriz de categorización; en este caso se elaboró teniendo en cuenta el cumplimiento o no de los indicadores seleccionados de los Objetivos del Desarrollo del Milenio relacionados con la salud, dentro de la operatividad en el enlace con la Atención Primaria en Salud. Posteriormente se realizó un análisis exhaustivo de diferentes variables que

fueron tabuladas obteniendo valores cualitativos y cuantitativos, los cuales fueron expresados en tablas y gráficas. Resultados: En contexto general de los países estudiados, la principal fortaleza de la aplicación de la estrategia de Atención Primaria en Salud, se encuentra en los ODM 5: Mejorar la Salud materna en el indicador de resultado de Tasa de mortalidad materna y en el

ODM 6: Combatir el VIH/SIDA, el paludismo y otras enfermedades en su indicador de resultado: Hombres y mujeres de 15 a 49 años que viven con VIH/SIDA; en el que más se evidencian falencias o impactos negativos como mayoría en 4 países: el ODM 8: Establecer una alianza mundial para el desarrollo, en su indicador de resultado: población con acceso estable a medicamentos esenciales a precios razonables.

\section{Citar como:}

Sepúlveda Correa, D., Montaña Vásquez, J. R., \& Vargas Guette, M. L. (2021). Comparación de los modelos de atención primaria en salud desde un enfoque sanitario en Colombia y sus países fronterizos. Movimiento Científico, 15 (1), [pgIn]-[pgOut]. Obtenido de: https:// revmovimientocientifico.ibero.edu.co/article/view/2218

Daniela Sepúlveda Correa, Esp od

Source | Filiacion:

Activos - Fiduprevisora

BIO:

Odontóloga egresada de la Universidad de Antioquia y especialista en Gerencia de la Calidad en Salud de la Corporación Universitaria Iberoamericana.

City | Ciudad:

Bello[co]

e-mail:

dasepulco@hotmail.com
José Rafael Montaña Vásquez, Esp Ft.

Source | Filiacion:

ECOOPSOSEPS

BIO:

Enfermero profesional egresado de la Universidad Nacional de Colombia y especialista en Gerencia de la Calidad en salud de la Corporación Universitaria Iberoamericana.

City | Ciudad:

Bogotá[co]

e-mail:

jose.montana.vasquez@gmail.com
Martha Liliana Vargas Guette Esp Enf.

Source | Filiacion:

Centros Hospitalarios del Caribe (Clínica CEHOCA)

$\mathrm{BIO}$

Enfermera profesional egresada de la Universidad del Magdalena y Especialista en Gerencia de la Calidad en Salud de la Corporación Universitaria Iberoamericana.

City | Ciudad:

Santa Marta[Co]

e-mail:

nursingmlvg@hotmail.com 


\section{Comparación de los modelos de atención primaria en salud desde un enfoque sanitario en Colombia y sus países fronterizos}

A comparison of primary health care models from a health approach in Colombia and its limiting countries

Daniela Sepúlveda Correa José Rafael Montaña Vásquez Martha Liliana Vargas Guette

\section{Introducción}

Según la Organización Mundial de la Salud (OMS), la Atención Primaria en Salud (APS) se define como: "La asistencia sanitaria esencial accesible a todos los individuos y familias de la comunidad a través de medios aceptables para ellos, con su plena participación y a un costo asequible para la comunidad y el país." (Organización Mundial de la Salud, s.f.)

La importancia de este concepto radica en las condiciones mínimas y necesarias para que, desde el aspecto sanitario, se mejore la calidad de vida de las personas. Se menciona además en esta definición, las palabras "participación” y “costo", situaciones que pueden variar según la cultura y la economía de cada país, lo que hace que sus políticas públicas sean adaptativas.

En este estudio se realizó la comparación los modelos de Atención Primaria en Salud de Colombia y sus países fronterizos con datos del año 2018, teniendo como base, indicadores de resultado de los Objetivos del Desarrollo del Milenio relacionados con la salud. La razón por la cual se decidió establecer la comparación con los ODM radica en que por su creación y estructura para el año 2000, se consideró que en los diversos países se debió crear una base importante para la formación de programas relacionados con la Atención Primaria en Salud por lo cual se establece que, desde luego, se podría correlacionar un impacto importante fomentado desde años atrás. Además, se tomaron datos del año 2018 ya que por la situación del COVID-19 y la pandemia pudieron existir limitantes en la calidad de los datos en los años posteriores. Se desarrolló una revisión de fuentes investigativas, páginas ministeriales y abaladas en salud de cada país y se realizó un análisis exhaustivo cuantitativo y cualitativo para definir los modelos que posiblemente pueden generar un impacto positivo para tomarse como referencia en los planes de mejora de cada país. Teniendo como base todo lo anterior, surge la siguiente pregunta: 


\section{Comparación de los modelos de atención primaria en salud desde un enfoque}

¿Por qué es relevante el estudio comparativo de los diferentes modelos de Atención Primaria en Salud en los países aledaños a Colombia, evidenciando la aplicación de la estrategia, revelando su impacto y detectando sus posibles barreras y factores facilitadores?

El tema de Atención Primaria en Salud, se encuentra ligado a una palabra: Desarrollo.

La mejor manera de sustentarlo está evidenciada en los Objetivos del Desarrollo del Milenio donde 6 de 8 objetivos, corresponden a un enfoque sanitario demostrando en muchas de sus metas e indicadores de resultado, el trabajo articulado de la promoción de la salud y la prevención de la enfermedad para poder alcanzarlos (Organización Mundial de la Salud, 2018).

Esta investigación, muestra de manera inicial una descripción detallada de su propósito además de la ejecución de una tarea metodológica conceptual minuciosa relacionada con la Atención Primaria en Salud y los Objetivos del desarrollo del Milenio; posteriormente, se contextualiza los temas relacionados con la situación actual de cada uno de los países en estudio: Panamá, Colombia, Venezuela, Brasil, Perú y Ecuador. Se ahonda en la profundidad del estudio de la aplicación de los diferentes programas de atención Primaria en Salud en los Objetivos del Desarrollo del Milenio relacionados con la salud y por último se ejecuta un análisis comparativo meticuloso sobre la situación de las temáticas entre los países, utilizando diferentes parámetros cuantitativos y cualitativos. Vale la pena aclarar que el término: "comparación", se entiende como un procedimiento de investigación que se basa en la diferenciación de conceptos, con el fin de establecer similitudes y diferencias entre los mismos.

Esta investigación consta en la comparación descriptiva, sin embargo, puede servir de base inicial para la promoción de un cambio en los modelos de Atención Primaria en Salud en los países elegidos para este análisis, teniendo en cuenta los posibles hallazgos y los aportes que pueden venir desde otros modelos

De esta manera, se busca que se beneficien diversos sectores:

Los gobiernos nacionales: en ellos, las entidades que son proactivas en la elaboración de políticas públicas en salud, esta investigación servirá como referencia para implementar modelos asertivos de Atención Primaria en Salud en cada país.

El sector salud: como base para modificar estrategias de promoción de la salud y prevención de la enfermedad para lograr un mayor impacto sanitario en la sociedad.

Las comunidades y pacientes: quienes se verán orientadas en su panorama actual y pueden tener criterios basados en la evidencia para hacer veeduría ciudadana a las entidades de salud, además de verse beneficiados por las pautas de mejora que adopten los entes gubernamentales para optimizar la estrategia de Atención Primaria en Salud.

Esta investigación también aportará datos suficientes que podrán dar a conocer la aplicación de la Atención Primaria en Salud dentro de los sistemas de salud de cada país desde un enfoque sanitario, que permitirá plantear estrategias de reforzamiento que contribuyan al desarrollo de las competencias y el porcentaje de cumplimiento de los Objetivos del Desarrollo del Milenio.

La Atención Primaria en Salud se ha establecido como el principal escenario de intervenciones para el progreso integral de la salud de la comunidad, disponiendo de un campo de conocimientos específico avalado por diferentes actores de los diversos sistemas de salud con funciones y procedimientos propios. La asistencia multidisciplinaria y la investigación son actividades habituales en la Atención Primaria en Salud, donde de forma particular la función investigativa se ocupa de aplicar el método científico en el proceso de identificación y solución de problemas de salud en la comunidad, con un enfoque clínico, epidemiológico y social, que en este caso, se tomó como base el enfoque sanitario, así como planificar y ejecutar investigaciones científicas en el campo de acción como profesionales de la salud, determinando los aspectos a mejorar en los modelos de Atención Primaria en Salud en los países objeto de estudio lo que permitirá una mejor implementación de estos. (Pérez, 2014)

Existe una amplia literatura que ha comparado los diferentes sistemas de salud de la región andina, el caribe, entre otras. Sin embargo, en la búsqueda bibliográfica, no se encuentran estudios que comparen los modelos de Atención Primaria en Salud de Colombia con los países fronterizos, por lo que representaría un aporte en relación con las variables que representan éxito o fracaso en estos modelos, lo cual permitiría más adelante poder presentar un plan de mejoramiento del mismo, teniendo en cuenta las experiencias y modelos actuales.

\section{Antecedentes}

Desde el aspecto global, todo surge por la declaración del Alma Ata desde el año 1978 dónde se llevó a cabo la definición de Atención Primaria en Salud. En este concepto se tuvo en cuenta que había necesidades sanitarias prioritarias y determinantes importantes de la salud, se dio interés a las poblaciones más vulnerables y se trató de llevar al desarrollo general a través de la vinculación de factores sociales y económicos con la participación activa de estos sectores para que el modelo tuviera: equidad en el acceso a la salud y la eficacia en la prestación de los servicios. Surge a partir de esto la necesidad de comprender que la prevención de la enfermedad es tan importante como la curación de la misma, sin embargo, se llevaron a cabo muchas malinterpretaciones; por ejemplo: el cuerpo médico pensaba que solo debía enfocarse en un primer nivel de atención, se atribuyó que lo "barato" es sinónimo de "mala calidad" y para la época también surgió una crisis económica importante que tuvo gran influencia sobre los recursos para la salud por la aparición de enfermedades como el VIH, la tuberculosis y el paludismo que conllevaron a priorizar la situación de emergencia. Por esta razón el lema "Salud para todos" para el año 2000, no se cumplió. Sin embargo, se dio importancia en la inclusión sanitaria en los Objetivos del Desarrollo del Milenio (ODM) donde se asumen nuevos compromisos con el fortalecimiento de los sistemas basados en la Atención Primaria en Salud (Chan, 2008)

Los Objetivos del Desarrollo del Milenio en total son 8; 6 de ellos están relacionados con la salud. Estos son: ODM1: Erradicar la pobreza extrema y el hambre, ODM4: Reducir la mortalidad de los menores de 5 años, ODM5: Mejorar la salud materna, ODM6: Combatir el VIH/SIDA, la malaria y otras enfermedades, ODM7: Garantizar la sostenibilidad del medio ambiente y ODM8: Fomentar una alianza mundial para el desarrollo. En ellos, se aplican los diversos modelos de Atención Primaria en Salud como estrategia para el cumplimiento de estos (Organización Mundial de la Salud, 2018).

Ahora desde el contexto de América latina, Puentes (2012) señala que, las estrategias de Atención Primaria en Salud que se han 
implementado mostraron ser de gran impacto para el mejoramiento de la atención en salud de la población. (pág. 23)

Según Giraldo (2015), las estrategias de Atención Primaria en Salud son la base de los sistemas de salud de una región determinada, ya que resultados científicos han demostrado que son la clave para lograr la efectividad de los sistemas de salud y que pueden adaptarse a los diversos contextos sociales culturales y económicos de los diversos países, por lo que los gobiernos latinoamericanos se han hecho el compromiso de renovarlas. (pág.391)

Tomando otros aspectos importantes que señala Almeida (2018), es que la implementación de la Atención Primaria en Salud en América latina ha traído varios beneficios en dónde se puede destacar el aumento de la cobertura en tratamientos retrovirales, se duplica el gasto per cápita en salud y hay un descenso de las tasas de mortalidad infantil materna y por causas evitables. Conjuntamente se presentan avances en cuanto a la política de cobertura en salud ya que han aumentado a más de 46 millones de latinoamericanos en los últimos 25 años. Asimismo, Una de los principios de la Atención Primaria en Salud está relacionada con la reducción de enfermedades crónicas y factores de riesgo a través de un modelo de salud preventivo en el que se sugiere realizar por lo menos una consulta en el año. Sin embargo, el promedio de registros de atención en varios países de América Latina solo se realiza alrededor de un 20\% de consultas preventivas. (págs.1-4)

Según Giovanella (2015), actualmente en América latina, se encuentran en marcha diferentes procesos de revitalización de los modelos de Atención Primaria en Salud. Su implementación es progresivay sin embargo todavía no se han alcanzado completamente los resultados esperados. (pág. 319).

Desde el contexto local en Colombia, en la ley 1438 de 2011, capítulo 3, articulo 12, la Atención Primaria en Salud es la estrategia de coordinación intersectorial que permite la atención integral e integrada, desde la salud pública, la promoción de la salud, la prevención de la enfermedad, el diagnóstico, tratamiento y la rehabilitación del paciente en todos los niveles de complejidad a fin de garantizar un mayor nivel de bienestar en los usuarios, sin perjuicio de las competencias legales de cada uno de los actores del Sistema General de Seguridad Social en Salud. Además, está constituida por 3 componentes que se integran y son interdependientes entre ellos: los servicios de salud, la acción intersectorial/transectorial y la participación social, comunitaria y ciudadana a través de métodos, tecnologías y prácticas fundamentadas en la ciencia para que contribuyan a la equidad, solidaridad y costo - efectividad de los servicios de salud. Para esto el Ministerio de Protección Social se encarga de definir e implementar las herramientas necesarias como los registros de salud electrónicos a nivel nacional entre otros instrumentos técnicos. (Congreso de Colombia, 2011)

De acuerdo con Padilla (2012) en su artículo reflexivo: "Análisis de la APS dentro de las políticas sanitarias en Colombia", hay posibilidad de alcanzar sus objetivos si se eliminan ciertos elementos: el predominio del lucro por encima de las necesidades de atención, la separación estructural entre atención individual y acciones colectivas para definir a quien corresponde el gasto y la pérdida de referente territorial derivada del aseguramiento individual. Con el fin de fortalecer el estado actual del modelo de APS, se requiere como mínimo la búsqueda de estrategias que permitan hacer las modificaciones necesarias para cumplir de forma correcta con sus filosofías. (pág.144).

\section{Metodología}

El tipo de estudio fue descriptivo, observacional, retrospectivo, transversal, y mixto.

La población y muestra consiste en Información obtenida a partir de fuentes investigativas e informativas directas desde los sistemas de información públicos y ministerios de salud de cada país (Colombia, Panamá, Venezuela, Brasil, Perú y Ecuador) donde se suministren datos de los Indicadores seleccionados de los Objetivos del Desarrollo del Milenio relacionados con la salud realizando la comparativa de la información obtenida de cada país para el año 2018.

Para el desarrollo de la investigación se tuvo en cuenta la conceptualización de los conceptos ODM y APS basados en la Organización Mundial de la Salud:

Los Objetivos de Desarrollo del Milenio de las Naciones Unidas son 8 objetivos que los 191 Estados Miembros de las Naciones Unidas acordaron tratar de alcanzar para 2015. La Declaración del Milenio de las Naciones Unidas, firmada en septiembre de 2000, compromete a los dirigentes mundiales a luchar contra la pobreza, el hambre, la enfermedad, el analfabetismo, la degradación del medio ambiente y la discriminación contra la mujer. Los Objetivos del Desarrollo del Milenio, determinantes de esa Declaración, tienen metas e indicadores específicos. (Organización Mundial de la Salud, s.f.)

La Atención Primaria de Salud es la asistencia sanitaria esencial accesible a todos los individuos y familias de la comunidad a través de medios aceptables para ellos, con su plena participación y a un costo asequible para la comunidad y el país. Es el núcleo del sistema de salud del país y forma parte integral del desarrollo socioeconómico general de la comunidad. (Organización Mundial de la Salud, s.f.)

Teniendo en cuenta los conceptos anteriores, se realiza una contextualización general y las características generales de la ejecución de la estrategia Atención Primaria en Salud en cada país en estudio:

\section{COLOMBIA:}

- Se ajusta al contexto político, económico y social del país.

- Está centrada en los individuos, las familias y las comunidades.

- Se encuentra orientada a establecer condiciones de materialización del goce efectivo del derecho a la salud y la reducción de inequidades, mediante la atención integral a la salud desde una perspectiva de determinantes sociales y económicos.

- No es solo una provisión sectorial de servicios de salud, sino la acción coordinada del Estado, las instituciones y la sociedad para el mejoramiento de la salud y la creación de un ambiente sano y saludable.

Hace énfasis en promoción de la salud. (Ministerio de Salud, s.f.).

\section{PANAMÁ:}

- No está enfocado exclusivamente al tratamiento de las enfermedades, sino a la promoción de la salud. 


\section{Comparación de los modelos de atención primaria en salud desde un enfoque}

- Se está trabajando para formar alianzas estratégicas para formar grupos organizados en las comunidades y comités de salud de Atención Primaria en Salud.

- Los grupos conformados tienen la misión de realizar actividades de promoción enfocados en el concepto de estilos de vida saludable, teniendo además una relación estrecha con las comunidades.

- Establece vínculos para realizar alianzas estratégicas con asociaciones o instituciones instaladas en la comunidad para lograr una intersectorialidad que trabaje conjuntamente por la promoción de la salud y la prevención de la enfermedad.

- Se incluyen estrategias que promueven la consulta del paciente, reciba una atención oportuna y de calidad realizada por un equipo capacitado y sensible ante las necesidades de salud de la población. (Herrera, 2019)

\section{VENEZUELA:}

- Está centrado en la participación comunitaria.

- Busca la ampliación de su cobertura y capacidad de atención.

- Pretende ser más que una extensión de los servicios ofertados buscando un mejoramiento integral de la calidad de vida de sus ciudadanos. (Bastidas, 2018, pág. 3)

\section{BRASIL:}

- Se basa en un Programa de Atención Familiar.

- Busca una amplia gama de servicios de salud al alcance de los hogares.

- Tiene un enfoque participativo contando con todos los miembros de la comunidad, incluyendo representantes de la iglesia, organizaciones no gubernamentales y escuelas. (Organización Panamericana de la Salud, 2017, pág. 1)

\section{PERÚ:}

- Hace énfasis en las acciones de prevención de la enfermedad y promoción de la salud en el primer nivel de atención.

- Promueve la identificación de factores de riesgo en el ámbito comunitario y se orienta a los paquetes de intervenciones.

- Redefine la integralidad con el cumplimiento de los paquetes de intervención.

- Crea un marco institucional que incentiva la mejoría de la calidad de los servicios. (Villanueva, 2017)

\section{ECUADOR:}

- Surge como respuesta a una situación que sea necesaria modificar o mejorar.

- Es pertinente y adecuada al contexto local en donde se implementa.

- Promociona un desarrollo comunitario, familiar o individual.

- Promueve la equidad con énfasis en los grupos en situación de vulnerabilidad.

- Es sostenible en el tiempo.

- Fomenta su réplica en una situación distinta, pero con condiciones similares.

- Es innovadora.

- Fomenta las acciones intersectoriales.
- Considera elementos de evaluación de resultados, retroalimentación de las acciones y reorganización de ellas a partir de lo aprendido.

- Promueve la generación de políticas públicas. (Organizacion Panamericana de la Salud, s.f.)

Para la recolección de la información se diseñó una matriz de categorización, teniendo en cuenta que las variables de comparación deben tener una clasificación; en este caso se hizo teniendo en cuenta el cumplimiento o no de los indicadores seleccionados de los Objetivos del Desarrollo del Milenio relacionados con la salud dentro de la operatividad afín con la Atención Primaria en Salud.

En la matriz de análisis se registró la información obtenida de los países para el año 2018 dentro de la recolección de datos evidenciando el cumplimiento o no de dichos indicadores determinando la comparación de la aplicación de la estrategia Atención Primaria en Salud de cada país y así determinar cuáles son los modelos más adecuados de aplicación. De cada Objetivo del Desarrollo del Milenio relacionado con la salud, se compararon los siguientes indicadores de resultado:

ODM1: Erradicar la pobreza extrema y el hambre

INDICADOR: Niños menores de 5 años de peso inferior al normal.

ODM4: Reducir la mortalidad infantil

INDICADOR: Tasa de mortalidad de niños menores de 5 años

ODM5: Mejorar la salud materna.

INDICADOR: Tasa de mortalidad materna.

ODM6: CombatirelVIH/SIDA, el paludismoyotras enfermedades.

INDICADOR: Hombres y mujeres de 15 a 49 años que viven con VIH/SIDA.

ODM7: Garantizar la sostenibilidad del medio ambiente

INDICADOR: Acceso sostenible a mejores fuentes de abastecimiento de agua, urbana y rural.

ODM8: Establecer una alianza mundial para el desarrollo.

INDICADOR: Población con acceso estable a medicamentos esenciales a precios razonables.

En el plan de análisis, teniendo en cuenta que la investigación es una revisión y una comparación, no es meramente estadístico, se presentaron los datos de manera narrativa y mediante estadísticas se hace una interpretación de los hallazgos y un análisis preciso acerca de cuál es el modelo de Atención Primaria en Salud que presenta mejores resultados en su aplicación realizando valoraciones cuantitativas y cualitativas.

Teniendo presentes las consideraciones éticas, según el Artículo 11 de la Res. 008430 de 1993 del Ministerio de Salud Colombiano, la clasificación de esta investigación es categoría A: Investigación Sin Riesgo. Es una investigación documental en la que no se realiza ninguna intervención o modificación en ningún individuo. No se aplican cuestionarios, entrevistas, revisión de historias clínicas y documentación de carácter confidencial. 


\section{Resultados}

Se realizaron diferentes análisis de carácter cuantitativo y cualitativo.

Respecto a la aplicación de la estrategia Atención Primaria en Salud en el indicador del Objetivo de desarrollo del milenio relacionado con la salud, se observó que en los países estudiados, la principal fortaleza de la aplicación de la estrategia de Atención Primaria en Salud, se encuentra en IOS ODM 5: Mejorar la Salud materna en el indicador de resultado de Tasa de mortalidad materna y en el ODM 6: Combatir el VIH/SIDA, el paludismo y otras enfermedades en su indicador de resultado: Hombres y mujeres de 15 a 49 años que viven con VIH/SIDA.

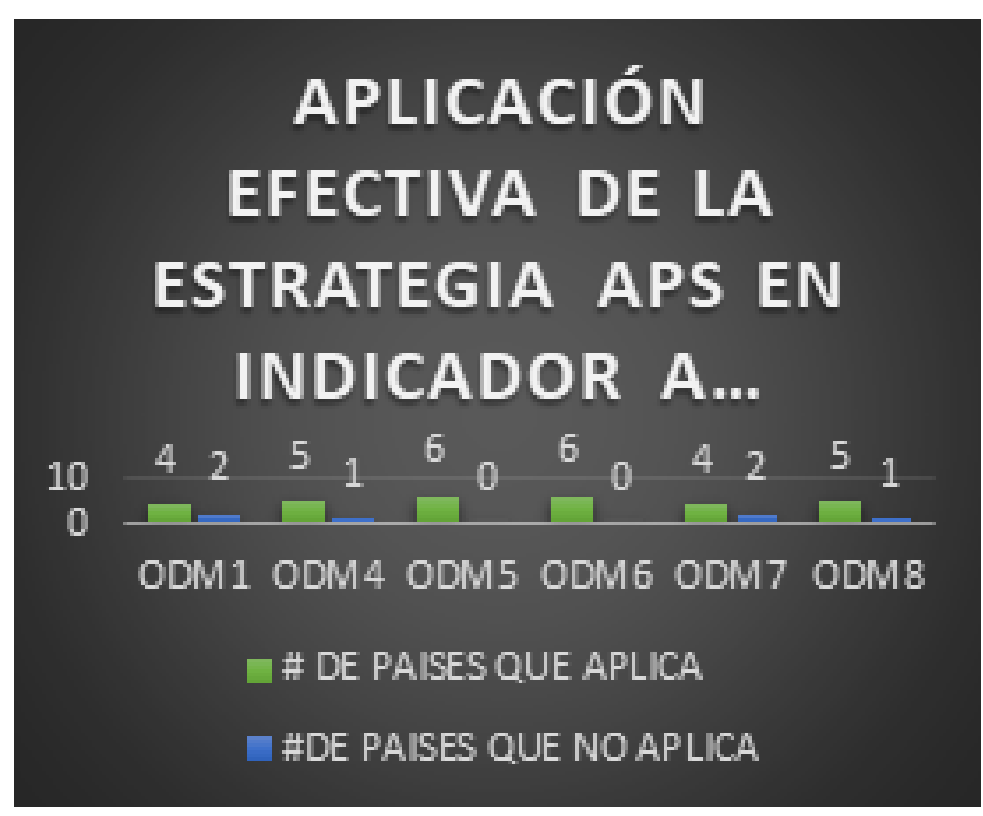

Respecto al impacto de la estrategia Atención Primaria en salud en el indicador del Objetivo del Desarrollo del Milenio relacionado con la salud se observó que, de los países estudiados, evaluando la aplicabilidad de los países en impacto positivo, se evidencia como fortaleza en 4 países el ODM 4: Reducir la mortalidad infantil en su indicador de resultado: Tasa de mortalidad de niños menores de 5 años. Y en el que más se evidencian falencias o impactos negativos como mayoría en 4 países: el ODM 8: Establecer una alianza mundial para el desarrollo, en su indicador de resultado: población con acceso estable a medicamentos esenciales a precios razonables.

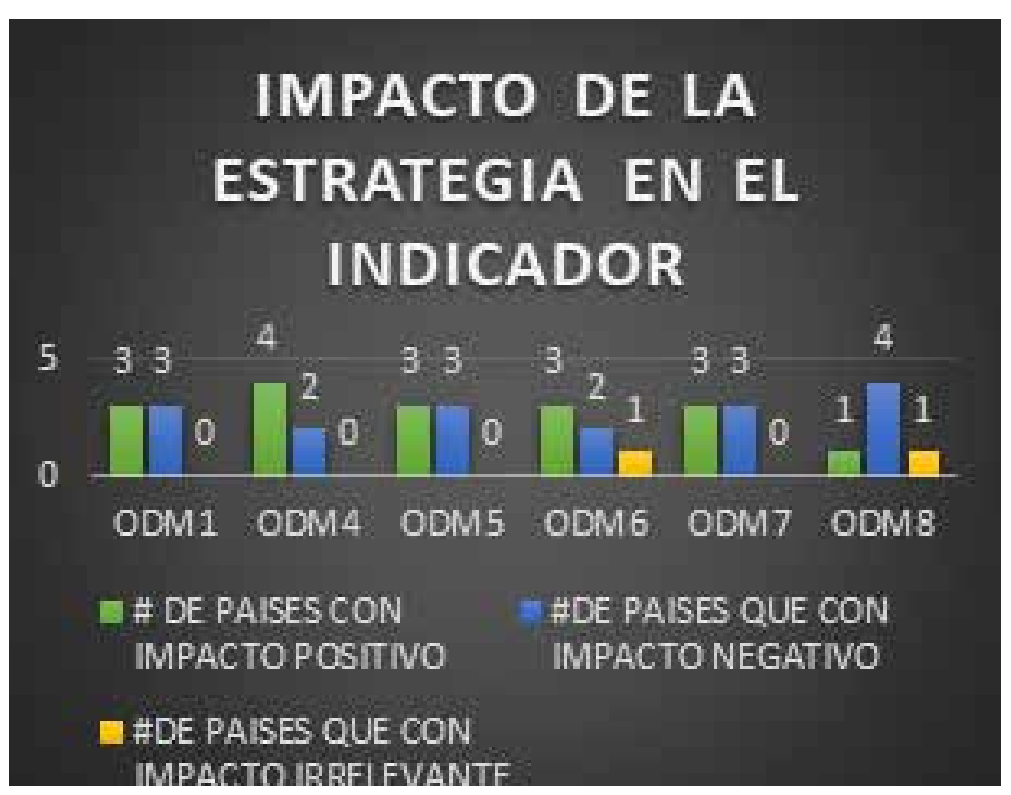

Respecto a las barreras detectadas en el alcance del cumplimiento del indicador del Objetivo del Desarrollo del Milenio relacionado con la Salud, todos los países tienen falencias que pueden ser representativas para el cumplimiento de los indicadores de resultado de los Objetivos del Desarrollo del Milenio relacionados con la salud.

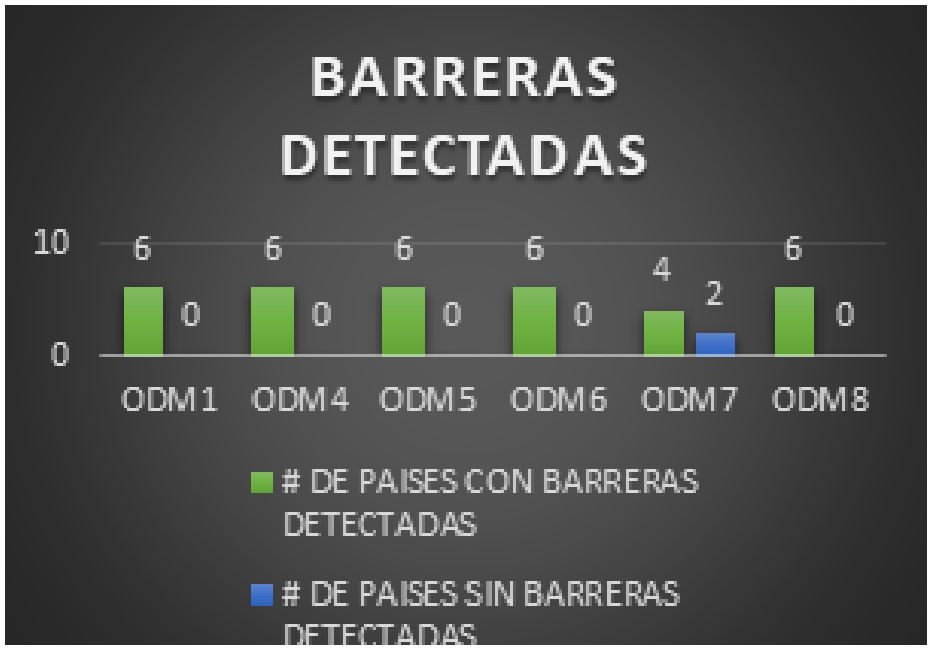

Respecto a los factores facilitadores en el alcance del cumplimiento del indicador del Objetivo del Desarrollo del Milenio relacionado con la salud, pese a que no todos los países cumplen con los indicadores de resultado de los Objetivos del Desarrollo del Milenio relacionados con la salud, todos presentan algunos factores facilitadores que pueden ser la iniciativa para el cumplimiento de los mismos.

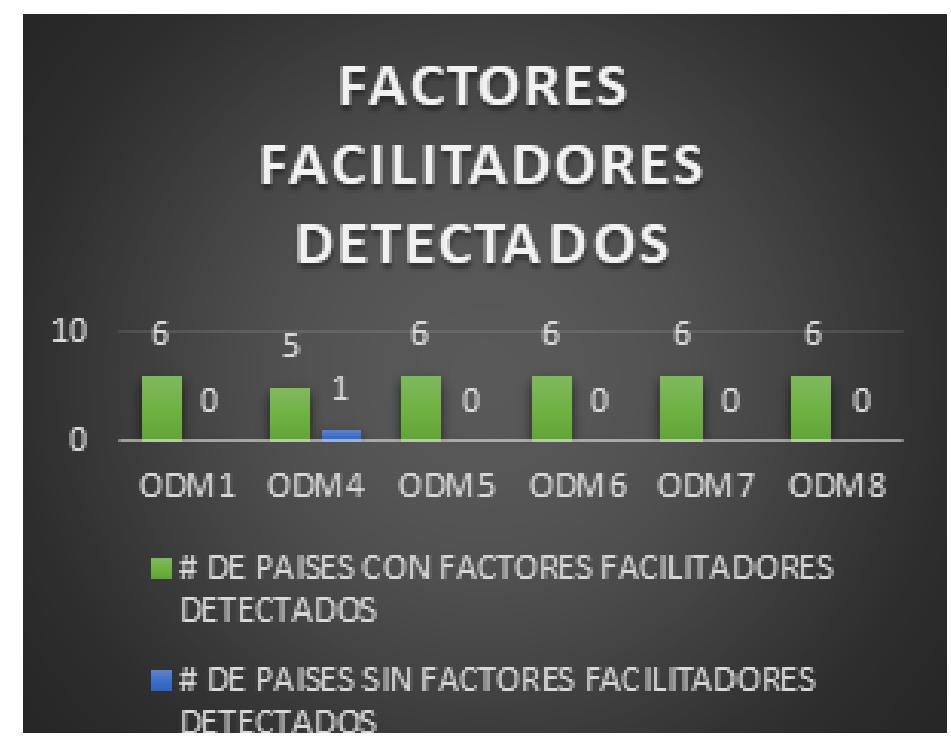

Respecto a la comparación de los países en el cumplimiento e impacto de la aplicación de la estrategia Atención Primaria en Salud en cada Objetivo del Desarrollo del Milenio relacionado con la salud, se asignaron unos valores cuantitativos para evaluar porcentualmente el cumplimiento e impacto de los indicadores de la siguiente manera:

SEGÚN SU APLICACIÓN: Aplica: 50\% - No aplica: 0\%

SEGÚN SU IMPACTO: Positivo: 50\% - Irrelevante: 25\% Negativo: 0\%

\section{Cumplimiento del ODM1en el indicador de resultado}

En los países analizados: Colombia, Panamá y Perú, tienen un cumplimiento del 100\% mientras Ecuador tiene el cumplimiento del $50 \%$ y Venezuela y Brasil no dan cumplimiento al indicador mencionado.

\section{Cumplimiento del ODM4 en el indicador de resultado}

En los países analizados: Panamá, Colombia, Brasily Perú, tienen un cumplimiento del 100\% mientras Ecuador tiene el cumplimiento del 50\% y Venezuela no da cumplimiento al indicador mencionado.

\section{Cumplimiento del ODM5 en el indicador de resultado}

En los países analizados: Colombia, Brasil y Perú, tienen un cumplimiento del 100\% mientras que Panamá, Venezuela y Ecuador tienen el cumplimiento del 50\%. 


\section{Cumplimiento del ODM6 en el indicador de resultado}

En los países analizados: Panamá, Colombia, Brasil y Perú, tienen un cumplimiento del 100\% mientras que Venezuela presenta un cumplimiento del 75\% y Ecuador tienen el cumplimiento del 50\%.

\section{Cumplimiento del ODM7 en el indicador de resultado}

En los países analizados: Panamá, Perú y Ecuador, tienen un cumplimiento del 100\% mientras que Colombia presenta un cumplimiento del 50\% y Venezuela y Brasil no dan cumplimiento al objetivo.

\section{Cumplimiento del ODM8 en el indicador de resultado}

En los países analizados: Brasil, tiene un cumplimiento del 100\% mientras que Panamá presenta un cumplimiento del 75\%, en Perú, Ecuador y Colombia el cumplimiento es del 50\% y en Venezuela no se da cumplimiento.

Respecto al comportamiento de los indicadores en cada país, en el cumplimiento e impacto de la aplicación de la estrategia Atención Primaria en Salud en cada Objetivo del Desarrollo del Milenio relacionado con la salud, se asignaron unos valores cuantitativos para evaluar porcentualmente el cumplimiento e impacto de los indicadores de la siguiente manera:

SEGÚN SU APLICACIÓN: Aplica: 50\% - No aplica: 0\%

SEGÚN SU IMPACTO: Positivo: 50\% - Irrelevante: 25\% Negativo: 0\%

\section{PANAMÁ}

Tiene fortalecidos sus programas de Atención Primaria en Salud en los indicadores de resultado de Objetivos del Desarrollo del Milenio relacionados con la salud: 1, 4, 6, Y 7 mientras que falta fortalecer el 5 y 8.

\section{COLOMBIA}

Tiene fortalecidos sus programas de Atención Primaria en Salud en los indicadores de resultado de Objetivos del Desarrollo del Milenio relacionados con la salud: 1, 4, Y 5 mientras que falta fortalecer el 6, 7 y 8.

\section{VENEZUELA}

Requiere un fortalecimiento en todos sus programas de Atención Primaria en Salud en los indicadores de resultado de Objetivos del Desarrollo del Milenio relacionados con la salud: 1, 4, 5, 6, 7 y 8.

\section{BRASIL}

Tiene fortalecidos sus programas de Atención Primaria en Salud en los indicadores de resultado de Objetivos del Desarrollo del Milenio relacionados con la salud: 4, 5 y 8 mientras que falta fortalecer el 1, 6 y 7 .

\section{PERÚ}

Tiene fortalecidos sus programas de Atención Primaria en Salud en los indicadores de resultado de Objetivos del Desarrollo del Milenio relacionados con la salud: 1, 4, 5, 6 y 7 mientras que falta fortalecer el 8.

\section{ECUADOR}

Tiene fortalecidos sus programas de Atención Primaria en Salud en el indicador de resultado del Objetivo del Desarrollo del Milenio relacionado con la salud: 7 , mientras que falta fortalecer el 1, 4, 5, 6 y 8.

\section{Discusión}

En todos los países se observa un fortalecimiento racional en los programas de Atención Primaria en Salud de los indicadores relacionados con la salud materna y con patologías de interés en salud pública como el VIH/ SIDA y paludismo.

La razón de ser del fortalecimiento de estos programas, está relacionada con que los aspectos anteriores pueden ser prevenibles en su gran medida. Cuando estos indicadores aumentan, están representados los impactos de los programas que se aplican en cada país.

Uno de los indicativos principales que permite verificar el índice de compromiso de los países con la reducción de mortalidad infantil se relaciona de forma directa con las políticas existentes encaminadas a la disminución de esta tasa, la disminución de enfermedades prevenibles durante la primera infancia orientadas desde la salud publica han demostrado ser efectivas y eficientes en este ítem especifico

Por otro lado, el acceso a medicamentos continúa siendo una gran barrera para la población general de países latinoamericanos, para los países objeto de esta investigación encontramos que en aquellos en los que hay políticas de acceso carecen de un control gubernamental de los precios por lo que se limita el acceso, mientras que aquellos en los que hay una política de control de los mismos son estas mismas políticas las que generan barreras administrativas que impiden que el acceso se dé en una forma oportuna y adecuada.

En relación con la política de medicamentos con la que medimos la eficacia de políticas para el ODM 8 vale la pena tomar el ejemplo del caso brasilero en donde tanto el acceso a medicamentos como su uso racional hace parte de las políticas estatales y en su componente básico se incluyen los medicamentos esenciales para el tratamiento de enfermedades prevenibles y su modelo no tiene comparación con ningún otro de los estudiados en la presente investigación.

Posterior al análisis de la información podemos concluir que, si bien todos los países del presente estudio tienen políticas encaminadas al cumplimiento de los objetivos ODM 4 y ODM8, es el primero sobre el cual existe mayor esfuerzo gubernamental en cuanto al cumplimiento de estas, en el acceso estable a medicamentos esenciales a precios razonables sólo Brasil establece un ejemplo a seguir. 
Todos los países estudiados presentan falencias y éstas se dan principalmente por una implementación inadecuada de los programas que se desarrollan para poder alcanzar cada Objetivo del Desarrollo del Milenio ya sea ésta por falta de cobertura en salud, vigilancia y control o por la falta de recursos económicos, ya a pesar de que se ha dado una reducción de los índices de pobreza, los recursos siguen siendo insuficiente.

Los Objetivos del Desarrollo del Milenio contribuyeron a que más de mil millones de persona mejoraran su situación económica, logrando la reducción de la pobreza extrema, combatir el hambre mejorando los accesos a la salud, por lo que la tasa de muertes de niños menores de 5 años y la mortalidad materna se disminuyeran, entre otros logros. No obstante, el avance ha sido desigual a través de las regiones y los países, lo que ha dejado enormes brechas.

Cada país representa en su medida el esmero por implementar programas para el cumplimiento de los Objetivos del Desarrollo del Milenio. El inconveniente no radica en que existan los programas, sino en la efectividad de éstos y el resultado que aporta en evidencia a los indicadores. La iniciativa es el recurso por el cual se construye la evolución y el impacto que generan los programas de Atención Primaria en Salud, pero se deben tener presentes las barreras que puedan representar una influencia sobre su desarrollo y ejecución lo que conlleva a programas exitosos o fallidos.

La iniciativa de los países en cumplir con los Objetivos del Desarrollo del Milenio es un paso fundamental para buscar el mejoramiento de los programas de Atención Primaria en Salud. Se sugiere que adopten modelos ejemplares para que puedan mitigar las barreras detectadas y promover y fortalecer los factores facilitadores.

Respecto al Objetivo del Desarrollo del Milenio 1, una de las principales formas de mejorar la calidad de vida y salud de las personas se relaciona con las estrategias encaminadas a la erradicación de la pobreza extrema y el hambre, en el análisis se evalúa cada ítem en niños menores de 5 años que presentan un peso inferior al percentil ideal para su edad, encontrando que dos de los países directamente no cumplen con aplicación de políticas de Atención Primaria en Salud dirigidas a la disminución del impacto del mismo, Venezuela debido a su crisis social y Brasil con los cambios que la derecha extrema ha implementado desde su acceso a la presidencia del país.

Si la idea de los Objetivos del Desarrollo del Milenio es lograr un desarrollo sostenible y que éste se evidencie en el mejoramiento de la calidad de vida y condiciones de salud de la población, es de vital importancia tomar el ejemplo de países como Panamá o Perú que, desde la implementación de programas y políticas encaminadas al cumplimiento de este indicador, logran un mejoramiento extensible a una gran parte del resto de la población.

Al analizar el Objetivo del Desarrollo del Milenio 4: Reducir la mortalidad infantil, en su indicador de resultado: Tasa de mortalidad de niños menores de 5 años Panamá, Colombia, Brasil y Perú son los países que mostraron mejores progresos en la salud de sus niños, al reducir notablemente todos los indicadores relacionados con la mortalidad en la niñez y por ende aumentar la esperanza de vida al nacer. Sin embargo, en Ecuador y Venezuela el panorama no es igual de alentador ya que no se logró este indicador en su totalidad lo que indica que las estrategias de Atención Primaria en Salud en estos 2 países no se han llevado de forma adecuada, además de esto existe subregistro de las defunciones lo cual indica que no se sabe a ciencia cierta cuál es la tasa de mortalidad infantil en estos.
La tasa de mortalidad de niños menores de cinco años se ha reducido a nivel mundial en más de la mitad, sin embargo, de los países estudiados dos de ellos que este caso son Ecuador y Venezuela en donde se ha dado un aumento de tasa de mortalidad infantil deben buscar políticas de fortalecimiento en la atención de la primera infancia con el fin de lograr un mejor desarrollo y avance en el objetivo y meta propuesta.

El análisis del Objetivo del Desarrollo del Milenio 5: Mejorar la salud Materna en el indicador de resultado: Tasa de mortalidad materna, se refleja en los países Colombia, Brasil y Perú por el interés en salud pública que le otorgan. En estos países se evidencia un decrecimiento en las cifras en el año 2018 comparado con años anteriores. Por el contrario, en los países Panamá, Venezuela y Ecuador, se observa el reflejo de un problema social importante donde la mortalidad materna continúa siendo un flagelo que no cesa. Existen muchas comunidades que requieren apoyo y que se encuentran olvidados por la lejanía, el fenómeno migratorio o la carencia de programas fortalecidos por la limitación de los recursos.

Respecto al Objetivo del Desarrollo del Milenio 6, desde los inicios de la pandemia del VIH, los países del mundo han implementado políticas de Atención Primaria en Salud encaminadas a controlar y hacer un seguimiento de los pacientes que en la actualidad viven con la enfermedad. Durante la investigación se encontró que, si bien todos los países estudiados cuentan con políticas encaminadas a la disminución de la prevalencia de la enfermedad en sus ciudadanos, no todas arrojan un resultado positivo, a esto debemos añadir la dificultad presentada con países como Venezuela en los que las cifras oficiales no son confiables o definitivamente no las comparten.

A pesar de que hay países con un cumplimiento del 100\% de las políticas encaminadas a la disminución de personas entre 15 y 49 años que viven con VIH-SIDA, el ejemplo de Brasil es uno de los que se deben tener en consideración, debido al éxito obtenido mediante su política de medicamentos al alcance de toda la población, pero también la posibilidad del uso de profilaxis preexposición y post exposición y la demarcación de la población no solo enfocada en los grupos que usualmente hacen parte de estas políticas, sino con trabajadoras sexuales, lo que representa un avance en el control de la pandemia.

El Objetivo del Desarrollo del Milenio 7, relacionado con la salud: Garantizar la sostenibilidad del medio ambiente, en su indicador de resultado: Acceso sostenible a mejores fuentes de abastecimiento de agua, urbana y rural, es uno de los objetivos que no ha presentado un alcance eficiente debido a que de los países estudiados solo el 50\% de estos garantiza un competo acceso a fuentes de abastecimiento de agua en donde se destaca Perú, al mantener una cobertura por encima del 98\% tanto para el área urbana como rural convirtiéndose en un abanderado en el logro de este objetivo, pero lastimosamente no todo es color de rosa porque en Colombia solo se cumple parcialmente con este objetivo y peor aún Venezuela y Brasil no dan cumplimiento a este, en el caso de estos dos países la falta de inversión en alcantarillado y acueducto en muy baja lo cual que hace que a pesar de tener buenos recursos hídricos, la población tenga desabastecimiento de agua potable.

Realizar control a través de los entes internacionales en donde se vigile la inversión que se hace de los presupuestos destinados para logar el cumplimiento de los Objetivo del Desarrollo del Milenio, que hoy en día se actualizaron a los Objetivos de Desarrollo Sostenible para poder garantizar el acceso a agua potable de los países que no dan cumplimiento a tan importante indicador. 


\section{Comparación de los modelos de atención primaria en salud desde un enfoque}

Se observa un cumplimiento muy variable en el Objetivo del Desarrollo del Milenio 8, relacionado con la salud: Establecer una alianza mundial para el desarrollo, en su indicador de resultado: Población con acceso estable a medicamentos esenciales a precios razonables. Como referente máximo se observa un programa muy fortalecido en Brasil ya que busca el favorecimiento sin exclusión al acceso farmacológico en los tratamientos médicos. La universalidad, la cobertura y la eficiencia podrían ser 3 conceptos que definen sus políticas de Atención Primaria en Salud relacionadas con el indicador de resultado. Sin embargo, se observa un incumplimiento categórico por parte del país Venezuela. Como se evidenció en la revisión bibliográfica, el problema político y social ha llevado a que la migración de esta población aumente por la limitación al acceso a medicamentos, buscando recuperar el derecho a la salud en otros países. Por otro lado, el cumplimiento parcial de Perú, Ecuador y Colombia, indican que requieren fortalecer sus programas relacionados.

El derecho a la salud es universal. No deberían existir limitaciones en el acceso a los tratamientos farmacológicos y deben estar implementados los programas de Atención Primaria en Salud en cuanto a la adopción de políticas públicas de educación en la regulación y control de la automedicación y de venta libre de fármacos.

\section{Conclusiones}

Se observaron los criterios de aplicación efectiva o no de la estrategia APS de cada país en estudio, además de describir su impacto, las barreras detectadas y los factores facilitadores llevando posteriormente a la identificación de los parámetros que pueden determinar que un modelo de APS sea exitoso o no.

En la actualidad existe mucha estigmatización cultural de los países los cuales pueden adquirir un señalamiento erróneo en sus sistemas. Quizás al leer el título de este texto, se pueden adquirir tentativamente unas conclusiones que al final de este texto conllevan a un cambio rotundo en el concepto personal.

En definitiva, cada modelo tiene una fortaleza especifica que podría servir como referente en cada país para poder lograr los objetivos en donde se ve altamente involucrado el desarrollo de cada país. Es entendible que existan factores externos como la política, la religión y la geografía, sin embargo, dentro de cada modelo, existe un criterio altamente moldeable a las situaciones siempre y cuando cada país busque cumplir con los parámetros mínimos para que un modelo de Atención Primaria en Salud, funcione.

También es importante resaltar que, entre todos los países en estudio, se crea un modelo de Atención Primaria en Salud altamente complementario: lo que para algunos países representa una barrera, en otros representan factores facilitadores. Por esta razón, establecer planes de mejora tomando como referentes a modelos exitosos, podrían generar un cambio benéfico para las naciones. Aunque en estudio solo se exponen 6 países, queda abierta una puerta a más estudios comparativos que vendrán a suplementar los modelos establecidos.

Como conclusiones generales de cada país en estudio, se evidencia lo siguiente:

Solamente Perú aplica políticas encaminadas al cumplimiento de estos indicadores y dichas políticas tienen un impacto positivo dentro del desarrollo de los objetivos en cada uno de ellos, siendo el país con mayor índice de cumplimiento.

Perú ha sido un país para tomar como ejemplo en cuanto al desarrollo de los Objetivos del Desarrollo del Milenio relacionados con la salud, mostrando gran un avance que se evidencia en el cumplimiento de 5 de las 6 metas propuestas.

Desde el gobierno de Perú se da inversión de recursos para logra alcanzar el desarrollo de los Objetivos Del Desarrollo Del Milenio en donde se prioriza la primera infancia, la desnutrición y la salud de las mujeres, no obstante se encuentran algunas barreras como lo son la poca cobertura en salud en las áreas rurales, restricción en el abastecimiento de medicamentos antirretrovirales e insuficiente provisión de medicamentos genéricos en los establecimientos públicos, por lo cual los pacientes compran de su bolsillo en farmacias privadas.

Se concluye además que la capacidad de vigilancia y control que ha dado Perú al desarrollo de los Objetivos Del Desarrollo De Milenio instaurando reglas obligatorias de formulación de presupuestos y creación de políticas que vincularon las asignaciones de recursos con resultados medibles se ven reflejados en los avances tan notorios al lograr un desarrollo positivo de las metas relacionadas con la salud casi en su totalidad.

Panamá cumple en el aspecto de tener políticas para cada uno de los indicadores, sin embargo, no todas representan un impacto positivo como en el caso de Perú.

Presenta fortalezas en 4 de los 6 Objetivos del Desarrollo del Milenio relacionados con la salud. En el análisis de sus indicadores de resultado se evidencia que tiene fortalecidos los programas para la prevención de enfermedades, la búsqueda de la erradicación de la pobreza extrema y el hambre y mejorar las fuentes de abastecimiento hídricas para el mejoramiento de la calidad devida de la población, sin embargo, se observa que falta cobertura de salud en las poblaciones más alejadas denominadas: "Comarcas" representa un gran riesgo para las gestantes y esto se refleja en los indicadores de mortalidad materna además de presentar grandes inconvenientes para el acceso y regulación de consumo de medicamentos. Las principales falencias están representadas en el sistema de salud en su cobertura y en el impacto de los programas que requieren un fortalecimiento especial en algunas poblaciones donde se refleja el olvido estatal.

Se concluye que Panamá tiene grandes fortalezas en el cumplimiento de los Objetivos del Desarrollo del Milenio relacionados con la salud, sin embargo, para mitigar las barreras detectadas en los indicadores de resultado que presentan debilidades, se hace importante un análisis de cobertura en el sistema de salud para poder llevar a cabo programas de Atención Primaria en Salud donde se logre un impacto positivo.

Ecuador, en el papel cumple con cada uno de los indicadores, sin embargo, cuando se revisó el impacto de estas políticas en los mismos, se evidenció que únicamente el objetivo 7 relacionado con el acceso sostenible a mejores fuentes de abastecimiento de agua, urbana y rural, tiene un impacto positivo en la población del país lo que afirma que no es suficiente contar con una política encaminada al cumplimiento, sino que requiere seguimiento de los resultados.

Ecuador presentó cifras importantes en cuanto a la mejoría de la salud infantil y materna sin embargo esto no fue suficiente para lograr alcanzar los Objetivos del Desarrollo del Milenio relacionados con salud y esto está dado por todas las barreras que se presentan para llegar a las metas propuestas dentro de estas se puede señalar: 
aumento de los índices de pobreza, población rural con poco acceso a alimentos y micronutrientes, aumento de la población migrante y falta de cobertura en acceso a servicios de salud.

El Objetivo del Desarrollo del Milenio 7: Garantizar la sostenibilidad del medio ambiente en su indicador: Acceso sostenible a mejores fuentes de abastecimiento de agua, urbana y rural, es el único con un desarrollo del 100\% en Ecuador y aunque este es importante no es suficiente para garantizar que las estrategias de Atención Primaria en Salud que se están llevando a cabo son las adecuadas.

Como conclusión, las políticas de Atención Primaria en Salud que se han instaurado en Ecuador, han sido ineficientes para la contribución al logro de los Objetivos del Desarrollo del Milenio ya que la revisión bibliográfica evidencia que, a pesar de tener programas bien constituidos, su impacto es negativo y las metas no fueron cumplidas.

Con Colombia se evidenció que, a nivel de políticas, únicamente el indicador 8 relacionado con el acceso estable a medicamentos esenciales a precios razonables, no cuenta con una política clara encaminada al mismo sin embargo, incluso algunos de los objetivos que cuentan con políticas encaminadas a su cumplimiento presentan un aspecto negativo de cara al ciudadano, como es el caso de la pandemia del VIH/SIDA cuyos resultados distan mucho de ser los esperados y el acceso a mejores fuentes de agua potable a nivel urbano y especialmente en las zonas rurales.

Colombia cumple de manera parcial los Objetivos del Desarrollo del Milenio relacionados con la salud ya que presenta fortalecidos los programas de Atención Primaria en Salud en 3 de 6 indicadores de resultados.

Se evidencia que existe una priorización por la salud infantil y materna además de la erradicación de la pobreza extremay el hambre. Sin embargo, se encuentran barreras en los programas relacionados con algunas enfermedades, la falta de cobertura y abastecimiento de agua potable y la falta de regulación farmacológica y el acceso a algunos medicamentos.

Lo anterior indica que Colombia tiene unos intereses sectorizados en la salud pública y por esta razón presenta un especial énfasis en algunos Objetivos del Desarrollo del Milenio dejando de lado la articulación de otros factores determinantes para mejorar la calidad de vida de la población en general.

Se concluye que Colombia requiere fortalecer todos sus programas de Atención Primaria en Salud, pero generando la misma importancia que requieren para poder alcanzar el desarrollo. La promoción de la salud y la prevención de la enfermedad son la base del impulso para poder alcanzar las metas en los Objetivos del Desarrollo del Milenio, sin embargo, factores agravantes como la migración no deberían entorpecer los procesos cuando se encuentran bien establecidos y estructurados.

El caso de Brasil es muy particular, ya que los objetivos a los que aplica políticas para el cumplimiento de los mismos tienen un impacto muy positivo, sin embargo en los objetivos 1 relacionado con la erradicación de la pobreza extrema y el hambrey el 7 que busca garantizar acceso sostenible a mejores fuentes de abastecimiento de agua no existen políticas definidas, Ilamando especialmente la atención este último objetivo debido a que el país concentra más del $12 \%$ de la totalidad de agua dulce del planeta siendo la reserva hídrica más importante y su descuido o daño producirían un impacto negativo importante a nivel mundial.
Brasil pone un énfasis especial en el cumplimiento de los objetivos del milenio relacionados con las gestantes y los menores de 5 años al igual que en el acceso a medicamentos de su población, sin embargo su compromiso a nivel de esfuerzos para erradicar la pobreza y el hambre, al igual que el acceso a agua potable requieren fortalecimiento, teniendo Brasil la reserva hídrica más grande del mundo, este compromiso no es únicamente con sus conciudadanos sino con el resto del planeta, a nivel del Objetivo del Desarrollo del Milenio 6 debemos tener en cuenta que Brasil ha sido uno de los países que más se ha afectado con la pandemia del VIH y aunque sus esfuerzos están bien encaminados, aún falta mucho camino por recorrer.

Se concluye que para que un país cumpla con la totalidad de los Objetivos del Desarrollo del Milenio y que las políticas de gobierno estén encaminadas al cumplimiento de los mismos requiere de un compromiso transversal, que trascienda los gobiernos y las ideologías políticas y que su meta sea alcanzar los objetivos, ya que al hacerlo se mejorará exponencialmente la condición de salud de las poblaciones y también la sostenibilidad ambiental.

En Venezuela fue donde se hallaron los mayores inconvenientes encuantoalainformacióndisponibleyaquelasituación políticaactual del país representa un hermetismo importante en el cumplimiento o no de los Objetivos del Desarrollo del Milenio relacionados con la salud en los indicadores específicos analizados, sin embargo, se logró identificar que, en cuanto a políticas, únicamente se cumplen los objetivos 5 y 6 pero teniendo un impacto negativo e irrelevante para cada uno de ellos. En relación al resto de estos, no se aplican políticas con el consecuente impacto negativo.

La actual situación socio política y la crisis humanitaria que sufre el vecino país hacen que el hermetismo en cuanto a cifras de cumplimiento sea el común denominador al intentar evaluar el cumplimiento o no de los Objetivos del Desarrollo del Milenio revisados en la investigación, sin embargo al intentar ahondar en los programas y políticas relacionadas con estos, se evidencia la politización de los mismos, tal es el caso como ejemplo del acceso a medicamentos esenciales, que para acceder a ellos se requiere un carnet ofertado por y para los simpatizantes del gobierno actual y aun así, no todas las veces se logra el acceso a los mismos.

Se concluye que la situación en salud de un país está directamente relacionada con las políticas que en este sentido establezcan sus gobiernos, por esta razón, la crisis política y humanitaria que actualmente aqueja al país vecino pone en evidencia esta premisa. Mientras que los gobiernos en un país se dediquen al saqueo de recursos, no habrá política sobre el papel que valga, siempre se pondrá en evidencia la verdadera situación a través de los testimonios de los propios ciudadanos.

\section{Referencias}

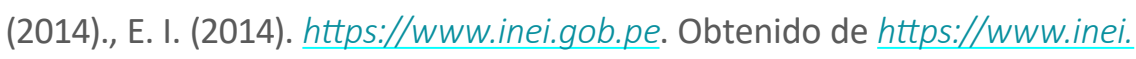
gob.pe/media/MenuRecursivo/publicaciones_digitales/Est/Lib1211/ pdf/cap013.pdf.

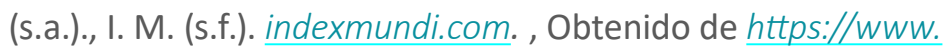
indexmundi.com/es/venezuela/tasa de mortalidad infantil. html\#: :text=Tasa\%20de\%20mortalidad\%20infantil\%20de\%20 Venezuela\&text=total\%3A\%2011\%2C9\%20muertes\%2F,1.000\%20 nacimientos\%20(2018\%20est.). 


\section{Comparación de los modelos de atención primaria en salud desde un enfoque}

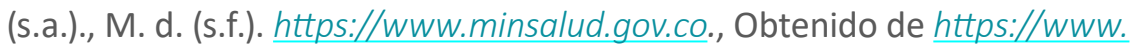
minsalud.gov.co/salud/Paginas/Atencion-primaria-en-salud.aspx.

(UNICEF), F. d. (2010). Servicios de atención para la primera infancia en Panamá. Panamá.

Acción Solidaria. (2021). Obtenido de https://accionsolidaria.info/envenezuela-la-mayor-cantidad-de-muertes-por-vih-sida-se-produceen-la-poblacion-de-entre-20-y-59-anos-il.

Alcantarillado, D. T. (2019). Estudio sectorial de los servicios públicos domiciliarios de acueducto y alcantarillado.

Alexandra Giraldo Osorio, C. V. (2013). La Atención Primaria de Salud: desafíos para su implementación en América Latina. Elsevier Doyma, 384-392.

Almeida Gisele, O. A. (2018). La atención primaria de salud en la Región de las Américas a 40 años de Alma-Ata. Panam Salud Publica, 1-6.

Álvarez, R. (2020). Aportes a la promoción de salud desde Barrio Adentro, Venezuela. Revista Cubana de Medicina General Integral, 2-3.

Anahp. (2018). Tras el aumento de la mortalidad infantil, el país puede volver al 'Mapa del Hambre. ANAHP. Obtenido de https://www. anahp.com.br/noticias/noticias-do-mercado/apos-aumento-demortalidade-infantil-pais-pode-voltar-para-mapa-da-fome/.

Araujo, J. (2016). https://dspace.unitru.edu.pe/. Obtenido de https:// dspace.unitru.edu.pe/bitstream/handle/UNITRU/15847/Araujo\%20 Gutierrez\%20Jacqueline\%20Denisse.pdf? sequence=1\&isAllowed $=y$.

Atun, I. (2015). Health-system reform and universal health coverage in Latin America. The Lancet.

Banco de desarrollo de America Latina. (2018). https://www.caf.com. Obtenido de https://www.caf.com/es/actualidad/noticias/2018/09/ promadec-mas-agua-mas-vida/.

Banco Mundial en América Latina y el Caribe. (18 de abril de 2018). https:// www.bancomundial.org/. Obtenido de $h$ ttps://www.bancomundial. org/es/results/2018/04/18/fighting-malnutrition-in-peru.

Bastidas, G. (2018). Atención primaria en salud. El caso Venezuela. Horizonte Sanitario. Obtenido de http://www.scielo.org. $m x / s c i e l o$. php?pid=S2007-74592018000300165\&script=sci_arttext.

Bastidas, G. (2018). Atención primaria en salud. El caso Venezuela. Horizonte Sanitario. . Volumen 17 Numero 3, 12.

Bengoa, F. (2018). Observatorio Venezolano de la Salud (OVS) y Red Agroalimentaria de Venezuela. Emergencia Humanitaria Compleja en Venezuela. Reporte Nacional, 1-6. , Obtenido de https://www. ovsalud.org/descargas/publica.

Bond, L. (2019). reliefweb. Obtenido de Unicef señala reducción histórica de la mortalidad infantil en Brasil.

Canto, Y. (2018). https://scielosp.org. Obtenido de $h t t p s: / / s c i e l o s p . o r g / p d f /$ rpsp/2020.v44/e27/es.

Carrillo Roa, A. (2018). Sistema de salud en Venezuela: cun paciente sin remedio? Sistema de salud en Venezuela: ¿un paciente sin remedio? 2 - 10. Obtenido de https://www.scielosp.org/article/ csp/2018.v34n3/e00058517/.

Castañeda Polania, M. V. (2017). Seguridad alimentaria, erradicación de la pobreza extrema en algunos países miembros del ALBA como Venezuela, Ecuador, Bolivia y Cuba: una visión extendida sobre las políticas y estrategias asumidas y orientadas, durante el periodo 2010 - 2013. Universidad de La Salle. Facultad de Ciencias Económicas y Sociales. , Economía, 15-16. Obtenido de https:// ciencia.lasalle.edu.co/cgi/viewcontent.cgi?

Castillo, J. (2018). www.iagua.es. Obtenido de https://www.iagua.es/blogs/ jesus-castillo/venezuela-modelo-gestion-servicio-agua-potablelimita-desarrollo-0.

Castro, A. (2019). https://www.unicef.org. Obtenido de https://www.unicef. org/ecuador/historias/agua-saneamiento-e-higiene-solucionespensadas-para-las-personas-y-la-naturaleza.

Cegonha., R. (2013). Fundacion Canguro. Obtenido de https:// fundacioncanguro.co/wp-content/uploads/2018/02/BRASIL-PauloBonilha.pdf.
Censos, I. N. (2019). https://www.ecuadorencifras.gob.ec. Obtenido de https://www.ecuadorencifras.gob.ec/documentos/web-inec/ Poblacion_y_Demografia/Nacimientos_Defunciones/2018/ Principales_resultados_nac_y_def_2018.pdf.

Centro de comunicación Nacional de Venezuela. (2021). Presidencia de Venezuela., Obtenido de https://presidenciave.com/parlamento/ joaquin-aguilar-es-una-politica-de-estado-del-regimen-mantenera-la-poblacion-padeciendo-por-la-falta-de-agua-en-el-pais/.

Chan, M. (15 de Septiembre de 2008). https://www.who.int/. Obtenido de https://www.who.int/dg/20080915/es/\#: :text=Hace\%2030\%20 a\%C3\%B1os\%2C\%20la\%20Declaraci\%C3\%B3n,sanitarias\%20 prioritarias\%20y\%20los\%20determinantes.

Chiliquinga, S. (2020). http://www.revsaludpublica.sld.cu. Obtenido de http://www.revsaludpublica.sld.cu/index.php/spu/article/ view/2029/1542.

Coello, C. (2018). https://www.edicionmedica.ec/. Obtenido de https:// www.edicionmedica.ec/secciones/salud-publica/el-vih-en-ecuadorpuede-ser-controlado-con-decision-politica-93095.

Colombia., C. d. (19 de Enero de 2011). POR MEDIO DE LA CUAL SE REFORMA EL SISTEMA GENERAL DE SEGURIDAD SOCIAL EN SALUD Y SE DICTAN OTRAS DISPOSICIONES. Ley 1438 de 2011. Bogotá, Cundinamarca, Colombia.

Cuba, M. S. (2018). http://www.scielo.org.pe. Obtenido de http://www.scielo.org.pe/scielo.php?pid=S1025$55832018000400013 \&$ script $=s c i$ arttext\&tlng $=p t$.

DaSilva, J. G. (2021). Por qué regresó Brasil al mapa del hambre? El País. Obtenido de $h$ ttps://elpais.com/planeta-futuro/2021-02-02/porque-regreso-brasil-al-mapa-del-hambre.html.

Delgado, D. (2020). Obtenido de https://www.recimundo.com/index.php/es/ article/view/1006/1659.

demografía., D. d. (2018). Análisis de situación de salud . ASIS. Bogotá.

demografía., D. d. (2019). Análisis de la situación de salud. ASIS. Bogota.

Díaz, A. (2019). El rol del profesional de enfermería en el sistema de Atención Primaria de Salud en Ecuador. . Revista Universitaria Ciencias y Tecnologia, 140-144. , Obtenido de https://uctunexpo. autanabooks.com/index.php/uct/article/view/231/344.

EFE., A. (2018). La mortalidad infantil crece en Brasil por primera vez desde 1990. Agencia EFE. Obtenido de https://www.efe.com/ efe/america/sociedad/la-mortalidad-infantil-crece-en-brasil-porprimera-vez-desde-1990/20000013-3693260.

Enriquez, Y. (2020). https://scielosp.org/., Obtenido de https://scielosp.org/ $p d f / r p s p / 2020 . v 44 / e 27 / e s$.

Español., C. e. (2019). CNN en Español. Obtenido de https://cnnespanol. cnn.com/2019/01/25/muertes-infantiles-aumentan-en-venezuelarevirtiendo-anos-en-mejoras-segun-estudio/.

Espinosa, V. (2017). https://www.scielosp.org. , Obtenido de https://www. scielosp.org/article/rpsp/2017.v41/e52/es/.

FAO, F. O. (2019). El estado de la seguridad alimentaria y la nutrición en el mundo 2019. Protegerse frente a la desaceleración y el debilitamiento de la economía.f. Obtenido de $h t t p: / / w w w . f a o$. org/3/ca5162es/ca5162es.pd.

Figueroa, V. (. (s.f.). http://metodos-avanzados.sociales.uba.ar. , Obtenido

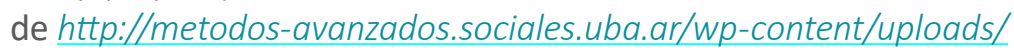
sites/216/2014/06/Presentaci\%C3\%B3n-de-Cais_final.pd.

Fuente, A. (2018). www.elpais.com. , Obtenido de https://elpais.com/ elpais/2018/04/23/planeta_futuro/1524502559_810295.html.

Galiani, S. (2017). Impacto de la estrategia de fortalecimiento de cobertura sobre la prestación de servicios de salud en Panamá. Banco Interamericano para el Desarrollo, 2.

Giovanella, L. F. (2015). Panorama de la Atención Primaria de Salud en Suramérica: concepciones, componentes y desafíos. Saúde em Debate, 300-322. 
Giraldo, Á. F. (2015). El rol de los profesionales de la salud en la Atención Primaria en Salud. . Revista Facultad Nacional de Salud Pública, $414-424$.

González, C. G. (2019). https://www.sciencedirect.com., Obtenido de $h$ ttps://www.sciencedirect.com/science/article/pii/ S1575181318300214\#!

Hernandez Herrera, M. (2018). Amenazas al bienestar de la infancia venezolana: Un reto para el pediatra ante una emergencia humanitaria compleja. Obtenido de https://docs.bvsalud.org/ biblioref/2019/11/1025917/art-3.pdf.

Herrera, L. G. (2018). La política nacional de medicamentos en el contexto de América Latina. Revista Cubana de Salud Pública. . Obtenido de http://scielo.sld.cu/scielo.php?script=sci arttext\&pid=S0864-34662018000200398.

lagua. (https://www.iagua.es. Obtenido de https://www.iagua.es/noticias/ epmaps-agua-quito/agua-quito-es-servicio-mejor-valoradoquitenos). 2018.

Informática., I. N. (2019). https://www.inei.gob.pe/., Obtenido de https:// www.inei.gob.pe/prensa/noticias/desnutricion-cronica-afectoal-122-de-la-poblacion-menor-de-cinco-anos-de-edad-en-elano-2018-11370.

Kauss, B. F. (2020). Reincidentes en el cuidado, pero sin derecho a la prevención: un análisis de la oferta de la profilaxis posexposición sexual al VIH en Porto Alegre, Brasil. . Obtenido de https://Www. scielosp.org/article/scol/2020.v16/e2463/es/.

Landaeta Jiménez Maritza, S. Y. (2018). Venezuela entre la inseguridad alimentaria y la malnutrición. . Anales Venezolanos de Nutricion, 70 - 75. , Obtenido de https://www.researchgate.net/profile/ Yaritza-Sifontes/publication/337472734_Venezuela_entre_la_inse.

Latina., C. B. (2018). Seguridad hídrica para evitar la escasez mundial de agua.

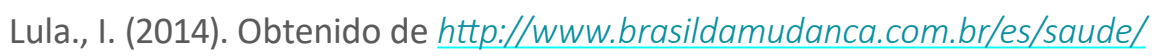
red-ciguena-da-asistencia-madre-y-bebe-en-el-embarazo-y-en-elparto.

macro, D. (2018). https://datosmacro.expansion.com/paises/panama. Obtenido de https://datosmacro.expansion.com/paises/panama.

Macro., D. (2021). Obtenido de https://datosmacro.expansion.com/paises/ brasil.

Marrero Juan Fernando, I. M. (2018). Lineamientos para legislar y justiciabilidad del derecho a la alimentacipon. . Observatorio del derecho a la alimentación en América Latina y el Caribe, 2 - 33. , Obtenido de $h \mathrm{ttp}: / / w w w . f a 0.0 \mathrm{rg} / 3 / \mathrm{i} 8936 \mathrm{es} /$ I8936ES.pdf.

Mundo., B. N. (2020). Hambre en Venezuela: los alarmantes niveles de desnutrición entre los niños venezolanos que se agravan por la pandemia. . BBC News. Obtenido de https://www.bbc.com/mundo/ noticias-america-latina-53381127.

Panamá., U. (2019). Situación de los derechos de la niñez y la adolescencia en Panamá. Panamá.

República de Panamá, m. d. (12 de Diciembre de 2018). Decreto Ejecutivo No 420. Panamá: Gageta oficial digital.

Restrepo-Espinosa, M. H. (22 de Diciembre de 2016). https://www. urosario.edu.co/., Obtenido de https://www.urosario.edu. co/Revista-Nova-Et-Vetera/Vol-2-Ed-22/Omnia/Origenes-ytrayectorias-de-la-atencion-primaria-en/\#: :text=La\%20APS\%20 se\%20origin\%C3\%B3\%20e.
RETEMA. (2018). La gran apuesta de Brasil ante la crisis hídrica. Obtenido de https://www.retema.es/noticia/la-gran-apuesta-de-brasil-antela-crisis-hidrica-2bnw5.

Rodriguez, T. (2018). https://lalineadefuego.info. , Obtenido de https:// lalineadefuego.info/2018/11/28/ecuador-pais-ineficiente-en-saludpublica-por-tomas-rodriguez-leon/.

salud, M. d. (2015-2020. Panamá). Plan Estratégico Nacional para la Reducción de la Morbilidad y Mortalidad Materna y Perinatal .

Salud., I. N. (2018). Boletin Epidemiológico semanal - Semana 42.

Salud., M. D. (2005). http://www.digemid.minsa.gob.pe. ,

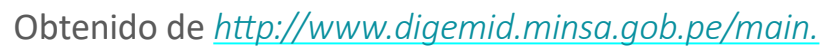
asp? Seccion $=603 \&$ Seccion Categoria $=22 \&$ pag $=3$.

salud., M. d. (2012). Dimensión Salud Ambiental. .

Salud., M. d. (2012-2021). Dimensión: sexualidad, derechos sexuales y reproductivos.

Télam. (2020). Sube el hambre en Brasil y afecta a 10,3 millones de personas. Telam. . Obtenido de https://www.telam.com.ar/ notas/202009/515370-subel-hambre--brasil-afecta--103-millones-personas.html\#: :text=09\%2F2020\%20indicadores-,Sube\%20 el\%20hambre\%20en.

Torrres, M. (2018). https://www.recimundo.com., Obtenido de https://www. recimundo.com/index.php/es/article/view/249/pdf.

Ugarte, O. (2019). http://www.scielo.org.pe., Obtenido de http://www. scielo.org.pe/pdf/afm/v80n1/a19v80n1.pdf.

Ulloa, O. (2020). http://repositorio.ug.edu.ec., Obtenido de http:// repositorio.ug.edu.ec/bitstream/redug/51024/1/ulloamu\%c3\%b1oz. pd.

UNICEF. (2018). Niños, niñas y adolescentes en Panamá, panorama . Panamá.

UNICEF. (2019). www.unicef.org. , Obtenido de https://www.unicef.org/lac/ comunicados-prensa/venezuela-m\%C3\%A1s-de-28-millones-depersonas-recibir\%C3\%A1n-mejor-acceso-agua-potable-con.

unidas., P. d. (2005). Objetivos de desarrollo del milenio: una mirada desde América Latina y el Caribe. Naciones Unidas.

Valdes, C. (2018). Encuesta de conocimientos y prácticas de la población relacionados al uso responsable de antibióticos. Ilaphar.

Vanguardia, L. (2020). Los contagios de VIH en Brasil cayeron un $7 \%$ en 2019. La Vanguardia. . Obtenido de https://www.lavanguardia. com/vida/20201201/49841887538/los-contagios-de-vih-en-brasilcayeron-un-7--en-2019.html.

Villanueva, R. (2017). http://cimfwonca.org. , Obtenido de Confederación

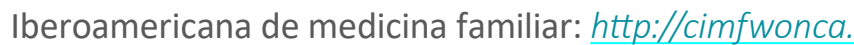
org/a-porposito-del-sistema-de-salud-peruano-reflexiones-de-lapresidenta-de-la-sopemfyc-rosa-villanueva/.

Watch., H. R. (2018). Venezuela: Las cifras evidencian una crisis de salud. . Obtenido de https://www.hrw.org/es/news/2018/11/15/venezuelalas-cifras-evidencian-una-crisis-de-salud.

Welle., D. (2019). Obtenido de https://www.dw.com/es/mortalidad-infantilen-venezuela-subi\%C3\%B3-40-por-ciento-en-ocho-a\%C3\%B1osseg\%C3\%BAn-estudio/a-47225316.

Welle., D. (2021). Obtenido de DW: https://www.dw.com/es/venezuelay-la-falta-de-agua-los-camiones-cisterna-son-una-estrategia-depropaganda-muy-cruel/a-53571000.

Welle., D. (2021). Vivir con sida en Venezuela. . Obtenido de https://www. dw.com/es/vivir-con-sida-en-venezuela/av-52113860. 